\title{
ketamine antidepressant actions in Electroconvulsive Therapy of Treatment-Resistant Depression
}

\section{J. Facucho-Oliveira' ${ }^{1}$, D. Esteves-Sousa ${ }^{1}$, B. Prates ${ }^{2}$, L. Roque ${ }^{2}$, F. Dinis ${ }^{2}$, R. Neves ${ }^{2}$, P. Varandas ${ }^{2}$. \\ ${ }^{1}$ Psychiatry and Mental Health Department, Hospital de Cascais, Lisbon}

${ }^{2}$ Electroconvulsive therapy unit, Casa de Saúde da Idanha - Instituto das Irmãs Hospitaleiras, Lisbon

Irmãs

Hospitaleiras

Casa de Saúde da Idanha

\section{BACKGROUND}

Major depressive disorder affects approximately $5 \%$ of the population with nearly 1-third of patients failing to achieve remission, despite adequate pharmacological treatment [1]. Although electroconvulsive therapy (ECT) is the gold standard approach for treatment resistant depression (TRD), a considerable fraction of patients also show a delayed improvement or appear to be resistant to ECT. One of the most promising rapid-acting antidepressants is ketamine, a noncompetitive $\mathrm{N}$ methyl-D-Aspartate receptor antagonist that can be used as an anesthetic during ECT to augment antidepressant efficacy [2].

Data on ECTs using ketamine are both scarce and conflicting, which lead the American Psychiatric Association to call for further studies [3].

An experimental, non-randomized clinical trial with consecutive sampling of TRD patient (persistant HAM-D $\geq 23$ ) was conducted. Patients who were initially assigned to 6 ECT sessions using thiopental anesthesia (Std ECT) but showed a very limited symptomatic relief were included in the study. Patients in Group 1 maintained treatment with Std ECT whereas patients in Group 2 were treated with further 6 ECT sessions using ketamine anesthesia (Ket ECT).

\section{INCLUSION CRITERIA AND STUDY DESIGN}

\section{Incluson Criteria:}

1) $\geq 3$-trials of antidepressants (TRD) and persistant HAM-D $\geq 23+$ Psychotic and/or Catatonic symptoms;

2) Patients treated with 6 Std ECTs with very limited or no improvement in HAM-D score and maintenance of Psychotic and/or Catatonic symptoms were assined to be included in Group 1 or Group 2.

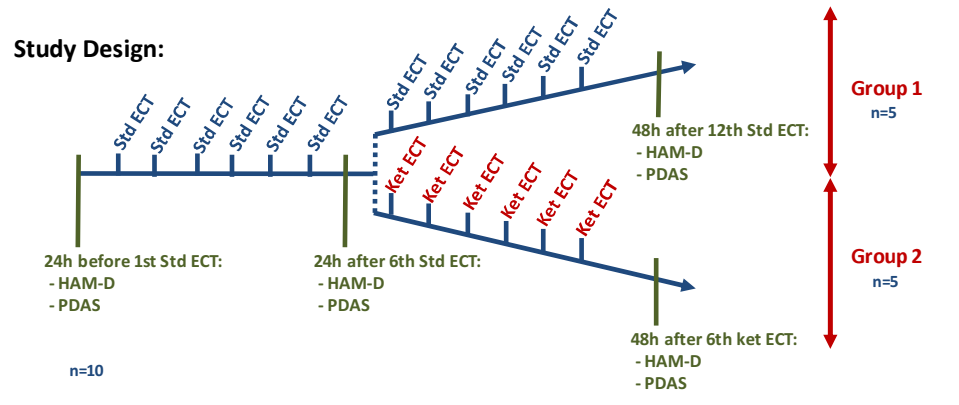

\section{METHODOLOGY}

Contraindications for ECT were assessed by anamnesis and complementary analytic and imagological testing. Depression was assessed using the Hamilton Depression Scale (HAM-D, 17-item version) and Psychotic Depression Assessment Scale (PDAS) 24 hours before performing Std ECT, 24 hours after the $6^{\text {th }}$ Std ECT and 48 hours after the last ECT session (Figure 1). Prescribed psychiatric medications were not discontinued.

ECT treatment was performed using a Thymatron System IV machine. Bilateral electrode placement was used and the stimulation parameters determined by the attending psychiatrist according to agebased methods and titrated in order to elicit seizures of a minimum of 25 seconds. ECT was conducted at a frequency of 2 sessions/week. Standard ECT sessions were conducted using standard anesthesia with thiopental. Patients enrolled for ECT sessions with ketamine, were treated with $5 \mathrm{mg}$ of haloperidol EV $\mathbf{3 0} \mathrm{min}$ before the procedure. Periprocedure management of hemodynamics and postanesthetic status were under discretion of the anesthesiologist.
Hospital) Cascais

\section{RESULTS}

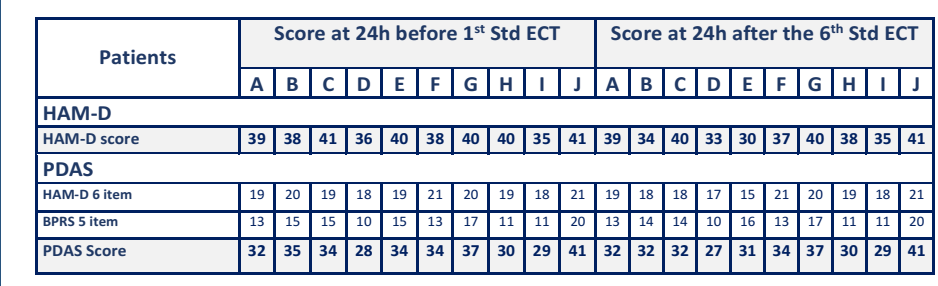

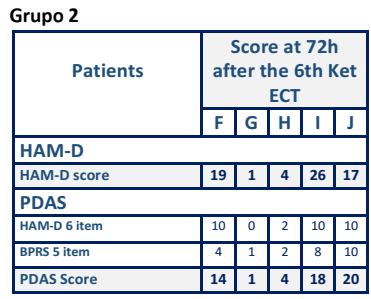

HAM-D

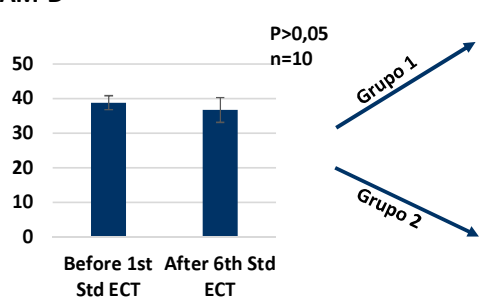

50

40

30

20

After 6th Std After 12th Std ECT ECT

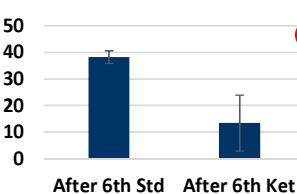

ECT ECT

ECT

PDAS
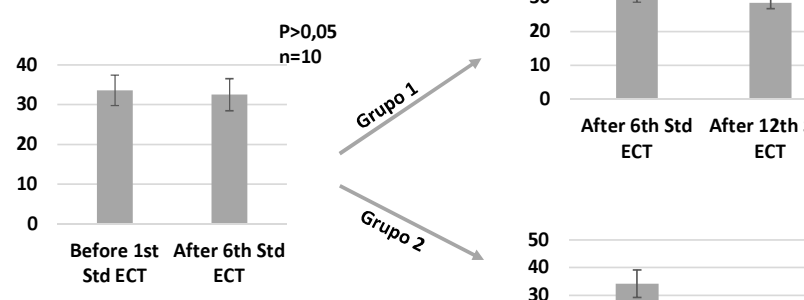

$P>0,05$

Ath Std After 12th Std

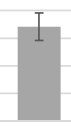

After 6th Std After 6th Ket

ECT ECT

\section{CONCLUSION}

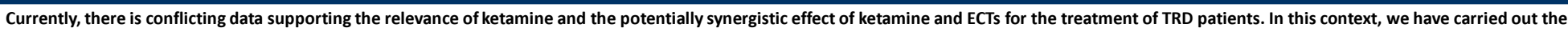

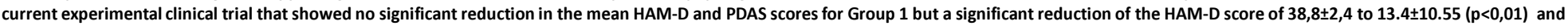

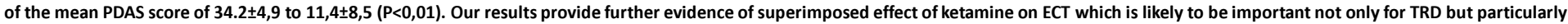
relevant for the subset of patients with TRD who appear to be resistant to ECT using standard methodology. 